DOI: https://doi.org/10.36910/6775-2524-0560-2021-42-02

УДК: 621.3 .061

Аракелян Варужан Павликович, к.т.н. доцент

Национальный Политехнический Университет Армении, Институт Энергетики и Электротехники, кафедра Электроэнергетика Республика Армения, г. Ереван

\title{
НОВЫЙ ПОДХОД К ОЦЕНКЕ ПОТЕРЬ МОЩНОСТИ ЭЛЕКТРОЭНЕРГЕТИЧЕСКОЙ СИСТЕМЫ С ПРИМЕНЕНИЕМ Z-МАТРИЦЫ
}

Аракелян В. П. Новый подход к оценке потери мощности электроэнергетической системы с применением Zматрицы. Вступительное слово о теме исследования: При исследовании режимов электроэнергетических систем различают параметры режима и схемы. Параметры схемы относятся к обобщенным параметрам сети. Узловая Z-матрица собственных и взаимных комплексных сопротивлений представляет собой матрицу обобщенных параметров. Параметры схемы системы делятся на активные и пассивные. Пассивные параметры - это продольные и поперечные параметры. Одним из важных технико- экономических показателей электрической системы является потери мощности. При исследовании режимов системы необходимо учитывать влияние продольных и поперечных пассивных параметров схемы на значения потерь. Цель научного исследования: Оценка потерь мощности в электроэнергетической системе с помощью Z-матрицы. Рассчитать потери, оценить изменение потерь активной и реактивной мощности обусловленный продольными и поперечными пассивными параметрами ,схемы системы. Описание научной и практической значимости работы: Научная ценность работы: Введен новый подход к оценке потерь мощности, основанный на новых формулах расчета коэффициентов потерь, применении Z-матрицы обобщенных параметров. Практическая значимость: обеспечивает универсальность для расчета, анализа и оценки потерь мощности в питающих электрических сетях. Описание методологии исследования: Учитывая разные виды моделирования линии электропередач современных электроэнергетических систем, сложность сетевых схем, теорию матриц, численные методы решения уравнений режима системы, коэффициенты потерь - B, с помощью Z-матрицы была получена новая формула для расчета, оценки и структурования потерь мощности. Основные результаты, выводы исследовательской работы: Исследование проводилось на макромодели электроэнергетической системы Армении. Анализ показывает, что предложенные формулы применимы к питающим электрическим сетям. Оцениваются изменение собственных активных и реактивных сопротивлений Z-матрицы , взаимных активных и реактивных сопротивлений Zматрицы, изменение коэффициентов потерь мощности обусловленный поперечными пассивными параметрами схемы системы. Ценность проведенного исследования (какой вклад данной работы в соответствующую отрасль знаний): Предложенный подход расширяет область применения обобщенных параметров при расчетах и анализе потерь мощности. Практическое значение итогов работы: Полученные новые формулы потерь мощностей позволяют комплексно проанализировать структуру потерь, выявить очаги, разработать организационно-технические меры по их снижению.

Ключевые слова: электроэнергетическая система, обобщенный параметр, поперечный параметр, продольный параметр, потери мощности, матрица, норма.

Аракелян В. П. Новий підхід до оцінки втрати потужності електроенергетичної системи з застосуванням Zматриці. Вступне слово про тему дослідження: При дослідженні режимів електроенергетичних систем розрізняють параметри режиму і схеми. Параметри схеми відносяться до узагальненими параметрами мережі. Вузлова Z-матриця власних і взаємних комплексних опорів $\epsilon$ матрицею узагальнених параметрів. Параметри схеми системи діляться на активні і пасивні. Пасивні параметри - це поздовжні і поперечні параметри. Одним з важливих техніко-економічних показників електричної системи $є$ втрати потужності. При дослідженні режимів системи необхідно враховувати вплив поздовжніх і поперечних пасивних параметрів схеми на значення втрат. Мета наукового дослідження: Оцінка втрат потужності в електроенергетичній системі за допомогою Z-матриці. Розрахувати втрати, оцінити зміна втрат активної та реактивної потужності обумовлений поздовжніми і поперечними пасивними параметрами, схеми системи. Опис наукової та практичної значущості роботи: Наукова цінність роботи: Введено новий підхід до оцінки втрат потужності, заснований на нових формулах розрахунку коефіцієнтів втрат, застосуванні Z-матриці узагальнених параметрів. Практична значимість: забезпечує універсальність для розрахунку, аналізу і оцінки втрат потужності в живильних електричних мережах. Опис методології дослідження: 3 огляду на різні види моделювання лінії електропередач сучасних електроенергетичних систем, складність мережевих схем, теорію матриць, чисельні методи рішення рівнянь режиму системи, коефіцієнти втрат - B, за допомогою Z-матриці була отримана нова формула для розрахунку, оцінки та структурованія втрат потужності . Основні результати, висновки дослідницької роботи: Дослідження проводилося на макромоделі електроенергетичної системи Вірменії. Аналіз показує, що запропоновані формули застосовні до живильних електричних мереж. Оцінюються зміна власних активних і реактивних опорів Z-матриці, взаємних активних і реактивних опорів Z-матриці, зміна коефіцієнтів втрат потужності обумовлений поперечними пасивними параметрами схеми системи. Цінність проведеного дослідження (який внесок даної роботи в відповідну галузь знань): Запропонований підхід розширює сферу застосування узагальнених параметрів при розрахунках і аналізі втрат потужності. Практичне значення результатів роботи: Отримані нові формули втрат потужностей дозволяють комплексно проаналізувати структуру втрат, виявити осередки, розробити організаційно-технічні заходи щодо їх зниження.

Ключові слова: електроенергетична система, узагальнений параметр, поперечний параметр, поздовжній параметр, втрати потужності, матриця, норма.

Arakelyan V. P. A new approach to estimating power losses of an electric power system using Z- matrix. Introductory speech on the research topic: When studying the modes of electric power systems, the parameters of the mode and the scheme are distinguished. Scheme parameters refer to generalized network parameters. The nodal Z-matrix of self and mutual impedances is a matrix of generalized parameters. System scheme parameters are divided into active and passive. Passive parameters are longitudinal and transverse parameters. One of the important technical and economic indexes of an electrical system is power losses. When studying 
the system modes, it is necessary to take into account the influence of the longitudinal and transverse passive parameters of the circuit on the loss values. The purpose of scientific research: Estimation of power losses of an electric power system using the Z-matrix. Calculate losses, assess the change in active and reactive power losses due to longitudinal and transverse passive parameters of the system circuit. Description of scientific and practical significance of the work: Scientific value of the work: A new approach to assessing power losses was introduced, based on new formulae for calculating loss factors, using the Z-matrix of generalized parameters. Practical value: provides versatility for calculating, analyzing and evaluating power losses in transmission electrical networks. Description of the research methodology: Taking into account the different types of power line modeling of modern electric power systems, the complexity of network schemes, matrix theory, numerical methods for solving system mode quations, loss factors - B, using the Zmatrix, a new formula was obtained for calculating, evaluating and structuring power losses. Main results, conclusions of the research work: The research was carried out on a macromodel of the electric power system of Armenia. The analysis shows that the proposed formulae are applicable to transmission electrical networks. The change in the self active and reactive resistances of the Z-matrix, the mutual active and reactive resistances of the Z-matrix, the change in the power loss factors due to the transverse passive parameters of the system circuit are evaluated. The value of the conducted research (what contribution of this work to the relevant branch of knowledge): The proposed approach expands the field of application of generalized parameters in the calculations and analysis of power losses. Practical significance of the results of work: The obtained new formulae for power losses allow for a comprehensive analysis of the structure of losses, identification of hot spots, and development of organizational and technical measures to reduce them.

Keywords: electric power system, generalized parameter, transverse parameter, longitudinal parameter, power losses, matrix, norm.

Постановка проблемы. Неравномерное распределение энергоресурсов в мире требует экономного использования ресурсов. Развитие современной электроэнергетической системы сопровождается дополнительными потерями мощности и снижением пропускной способности сети [1].С этой точки зрения актуальными становятся качественно новые подходы к расчету потерь мощности и структурированию. Необходимо оценить величину потерь как показатель эффективной работы системы, проанализировать ее структуру, выявить очаги, определить влияние обобщенных параметров сети, разработать организационные и технические мероприятия по снижению потерь, провести их нормирование. В настоящее время отсутствуют достаточно обоснованные формулы для определения структуры потерь по пассивной параметрам сети.

Анализ последних исследований и публикаций. Для расчета потерь мощности в электроэнергетических системах широко используются следующие методы и формулы [2-8].

Метод- $I^{2} \cdot R$.

$\Pi_{P}=3 \cdot I^{2} \cdot R$.

гдеІ-значение тока элемента системы,

$R$-активное сопротивление элемента системы.

Метод В-коэффициентов потерь.

Простая формула:

$\Pi_{P}=\sum_{i=1}^{M} \sum_{j=1}^{M} P_{i} \cdot B_{i j} \cdot P_{j}$.

Формула потерь Крона:

$\Pi_{P}=\sum_{i=1}^{M} \sum_{j=1}^{M} P_{i} \cdot B_{i j} \cdot P_{j}+\sum_{j=1}^{M} B_{0 j} \cdot P_{j}+B_{00}$.

где $P_{i}, P_{j}$-активные мощности і и ј узлов электроэнергетической системы соответственно,

$B_{i j}, B_{0 j}, B_{00}$-коэффициенты потерь или В-коэффициенты.

Метод разницы генерации и нагрузок.

$\Pi_{P}=\sum_{i=1}^{M+1} P_{\Gamma i}-\sum_{j=1}^{k} P_{\mathrm{H} j}$.

где $P_{\Gamma i}$ - активная мощность і-го генерации,

$P_{\mathrm{H} j}$ - активная мощность ј -го нагрузки.

Метод расчета перетоков в ЛЭП.

$\Pi_{S}=\dot{S_{l \jmath}}+\dot{S_{\jmath l}}$.

где $\dot{S_{l \jmath}}, \dot{S_{\jmath l}}$-мощности начала и конца линии соответственно.

Формулы связи узловых токов и пассивных параметров системы.

$\Pi_{P}=\sum_{i=1}^{M} \sum_{j=1}^{M}\left[\left(I_{p i} \cdot I_{p j}+I_{q i} \cdot I_{q j}\right) \cdot R_{i j}\right]$.

$\Pi_{Q}=\sum_{i=1}^{M} \sum_{j=1}^{M}\left[\left(I_{p i} \cdot I_{p j}+I_{q i} \cdot I_{q j}\right) \cdot X_{i j}\right]$.

где $I_{p i}, I_{p j}$-действительные части узловых комплексных токов,

$I_{q i}, I_{q j}-$ мнимые части узловых комплексных токов,

$R_{i j}, X_{i j}$ - активные и реактивные сопротивления линии.

Структурировать потерь по параметрам пассивной сети с помощью формул представленных методов расчета потерь невозможно. Для этого необходимо усовершенствовать формулы расчета потерь с учетом Z-матрицы обобщенных параметров схемы сети. 
Цель исследования. Для оценки потерь мощности предлагается использовать Z-матрицу обобщенных параметров схемы электроэнергетической системы (ЭЭС) с учетом:

1.Продольные пассивные параметры линий электропередач: активные и реактивные сопротивления.

2.Продольные и поперечные пассивные параметры линий электропередач: активные и реактивные сопротивления, активные и реактивные проводимости.

3. Новые формулы для расчета потерь.

Основной материал. Предположим, что электроэнергетическая система (ЭЭС) состоит из М+1 узлов (см. рис. 1.).

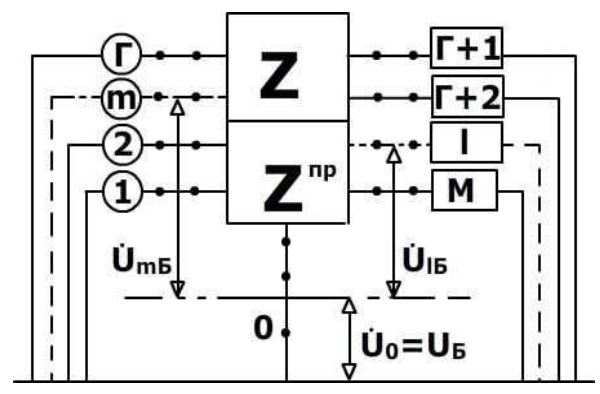

Рис. 1. Схема замещения ЭЭС Z-формой

В качестве базисного (балансного) выбирается узел с индексом “0”.Принимается, что мощности узлов станций $(1,2, \ldots, \Gamma)$ и нагрузки $(\Gamma+1, \Gamma+2, \ldots, \Gamma+\mathrm{H}=\mathrm{M})$ приведены на шины повышающих и понижающих трансформаторных подстанций. При этом уравнении состояния электрической системы в Z-форме принимает следующий вид [9]:

$$
\begin{aligned}
& \dot{U}_{1}=\dot{U}_{0 \mathrm{5}}+Z_{11} \cdot \dot{I}_{1}+Z_{12} \cdot \dot{I}_{2}+\cdots+Z_{1 M} \cdot \dot{I}_{M}, \\
& \dot{U}_{2}=\dot{U}_{0 \mathrm{~b}}+Z_{21} \cdot \dot{I}_{1}+Z_{22} \cdot \dot{I}_{2}+\cdots+Z_{2 M} \cdot \dot{I}_{M} \text {, } \\
& \begin{array}{c}
U_{M}=\dot{U}_{05}+Z_{M 1} \cdot \dot{I}_{1}+Z_{M 2} \cdot \dot{I}_{M}+\cdots+Z_{M M} \cdot \dot{I}_{M} \\
\dot{U}_{2}
\end{array}
\end{aligned}
$$

где $\dot{U}_{0 \mathrm{5}}, \dot{U}_{1}, \dot{U}_{2}, \ldots, \dot{U}_{M}$ - комплексные напряжении узлов $0,1, \ldots$, М электрической системы;

$\dot{I}_{1}, \dot{I}_{2}, \ldots, \dot{I}_{M}$ - комплексные токи узлов $1,2, \ldots, \mathrm{M}$ электрической системы;

$Z_{12}, \ldots, Z_{1 M}, Z_{21}, \ldots, Z_{2 M}, \ldots, Z_{M 1}, \ldots$-взаимные комплексные сопротивления независимых узлов электрической системы;

$Z_{11}, Z_{22}, \ldots, Z_{M M}$ - собственные комплексные сопротивления независимых узлов $1,2, \ldots, \mathrm{M}$

электрической системы.

Система алгебраических уравнений установившегося режима электрической системы (8) в компактной форме, принимает вид:

$\dot{U}=\dot{U}_{0 \mathrm{~b}}+Z \cdot \dot{I} \cdot(9)$

где $\dot{U}_{05}-$ вектор-столбец, каждый элемент которого равен напряжению базисного узла,

$\dot{U}$ и $\dot{I}$-вектор-столбцы узловых комплексных напряжений и токов,

$Z$-узловая комплексная матрица собственных и взаимных сопротивлений, обусловленный продольными и поперечными пассивными параметрами линий электропередач.

Система уравнений (9) представлена следующим образом:

$\dot{U}_{l}=U_{0 \text { Б }}+\sum_{j=1}^{M} Z_{i j} \cdot \dot{I}_{\jmath}, i=1,2, \ldots, M$.

После некоторых изменений, (10) представим в виде полной мощности:

$\dot{S}_{l}=U_{0 \text { Б }} \cdot \stackrel{*}{I_{i}}+\sum_{j=1}^{M} \stackrel{*}{I_{i}} \cdot Z_{i j} \cdot \stackrel{*}{I_{j}}, \quad i=1,2, \ldots, M$.

Подставляя выражения соответствующих параметров, разделяя действительные и мнимые части, для активной и реактивной мощности получаем:

$$
\begin{aligned}
& P_{i}=U_{0 \mathrm{~b}} \cdot I_{p i}+\sum_{j=1}^{M}\left[\left(I_{p i} \cdot I_{p j}+I_{q i} \cdot I_{q j}\right) \cdot R_{i j}-\left(I_{p i} \cdot I_{q j}-I_{q i} \cdot I_{p j}\right) \cdot X_{i j}\right], \mathrm{i}=1,2, \ldots \mathrm{M} . \\
& Q_{i}=-U_{0 \mathrm{~b}} \cdot I_{q i}+\sum_{j=1}^{M}\left[\left(I_{p i} \cdot I_{p j}+I_{q i} \cdot I_{q j}\right) \cdot X_{i j}+\left(I_{p i} \cdot I_{q j}-I_{q i} \cdot I_{p j}\right) \cdot R_{i j}\right], \mathrm{i}=1,2, \ldots \mathrm{M} .
\end{aligned}
$$

где $R_{i j}, X_{i j}$-активные и реактивные сопротивления линии обусловленные продольными и поперечными пассивными параметрами. 
Активные и реактивные мощности базисного узла системы определяются по формулам:

$P_{0 \mathrm{~b}}=-U_{0 \mathrm{~b}} \cdot \sum_{i=1}^{M} I_{p i}$.

$Q_{0 \mathrm{~B}}=-U_{0 \mathrm{~B}} \cdot \sum_{i=1}^{M} I_{q i}$

Из уравнений баланса мощности электроэнергетической системы, потери активной и реактивной мощности определяются следующими выражениями:

$\Pi_{P}=\sum_{i=1}^{M} P_{i}+P_{0 Б}$
$\Pi_{Q}=\sum_{i=1}^{M} Q_{i}+Q_{0 Б}$

Подставляя формулы (12) (14) в выражение (16), для потерь активной мощности получаем:

$\Pi_{P}=\sum_{i=1}^{M} \sum_{j=1}^{M}\left[\left(I_{p i} \cdot I_{p j}+I_{q i} \cdot I_{q j}\right) \cdot R_{i j}\right]$.

Подставляя формулы (13) l (15) в выражение (17), для потерь реактивной мощности получаем:

$\Pi_{Q}=\sum_{i=1}^{M} \sum_{j=1}^{M}\left[\left(I_{p i} \cdot I_{p j}+I_{q i} \cdot I_{q j}\right) \cdot X_{i j}\right]$.

В уравнениях (18) (19), заменяя компоненты тока узловыми мощностями, модулем и аргументом напряжения, получаем [10].

$\Pi_{P}=\sum_{i=1}^{M} \sum_{j=1}^{M}\left\{\frac{R_{i j}}{U_{i} \cdot U_{j}} \cdot\left[\left(P_{i} \cdot P_{j}+Q_{i} \cdot Q_{j}\right) \cdot \cos \left(\psi_{u i}-\psi_{u j}\right)+\left(P_{j} \cdot Q_{i}-P_{i} \cdot Q_{j}\right) \cdot \sin \left(\psi_{u i}-\psi_{u j}\right)\right]\right\}$.
$\Pi_{Q}=\sum_{i=1}^{M} \sum_{j=1}^{M}\left\{\frac{X_{i j}}{U_{i} \cdot U_{j}} \cdot\left[\left(P_{i} \cdot P_{j}+Q_{i} \cdot Q_{j}\right) \cdot \cos \left(\psi_{u i}-\psi_{u j}\right)+\left(P_{j} \cdot Q_{i}-P_{i} \cdot Q_{j}\right) \cdot \sin \left(\psi_{u i}-\psi_{u j}\right)\right]\right\}$.

где $U_{i}, U_{j}$-соответственно модули напряжений узлов і и ј системы,

$Q_{i}, Q_{j}$ - соответственно реактивные мощности узлов і и ј системы,

$\psi_{u i}, \psi_{u j}$ - соответственно аргументы напряжений узлов і и ј системы,

$R_{i j}$-действительная часть (активные сопротивления) $Z$-матрицы,

$X_{i j}$-мнимая часть (реактивные сопротивления) $Z$-матрицы.

Введем следующее обозначение:

$K_{i j}=\frac{1}{U_{i} \cdot U_{j}} \cdot\left[\left(P_{i} \cdot P_{j}+Q_{i} \cdot Q_{j}\right) \cdot \cos \left(\psi_{u i}-\psi_{u j}\right)+\left(P_{j} \cdot Q_{i}-P_{i} \cdot Q_{j}\right) \cdot \sin \left(\psi_{u i}-\psi_{u j}\right)\right]$.

Принимая во внимание обозначения (22), формулы (20) и (21) принимают следующие формы:

$\Pi_{P}=\sum_{i=1}^{M} \sum_{j=1}^{M} K_{i j} \cdot R_{i j}$.

$\Pi_{Q}=\sum_{i=1}^{M} \sum_{j=1}^{M} K_{i j} \cdot X_{i j}$.

где $K_{i j}$-называется коэффициентом потерь обусловленный продольными и поперечными пассивными параметрами линии.

В электрической системе с учетом только пассивных продольных параметров линий электропередач, уравнения (23) и (24) будут иметь следующие виды:

$\Pi_{P}^{\Pi p}=\sum_{i=1}^{M} \sum_{j=1}^{M} K_{i j}^{\Pi p} \cdot R_{i j}^{\Pi p}$.

$\Pi_{Q}^{\Pi p}=\sum_{i=1}^{M} \sum_{j=1}^{M} K_{i j}^{\Pi p} \cdot X_{i j}^{\Pi p}$.

Где $K_{i j}^{\text {пр }}$-называется коэффициентом потер обусловленный продольными пассивными параметрами линии,

$R_{i j}^{\text {пр }}, \quad X_{i j}^{\text {пр }}$-соответственно активные и реактивные сопротивления Z-матрицы обусловлены продольными пассивными параметрами схемы системы.

Оценим изменение Z-матрицы. Запишем вектор комплексных собственных сопротивлений Zматрицы, обусловленных продольными и поперечными пассивными параметрами линии:

$Z_{\text {соб }}=\operatorname{diag}(Z)=\left[Z_{11}, Z_{22}, \ldots, Z_{M M}\right] . \quad$ (27)

Вектор собственных комплексных сопротивлений Z-матрицы, обусловленных продольными пассивными параметрами линии, будет:

$Z_{\text {соб }}^{\text {пр }}=\operatorname{diag}\left(Z^{\text {пр }}\right)=\left[Z_{11}^{\text {пр }}, Z_{22}^{\text {пр }}, \ldots, Z_{M M}^{\text {пр }}\right] . \quad(28)$

Векторы взаимных комплексных сопротивлений Z-матрицы, будут:

$Z_{\text {в3 }}=\left[Z_{12}, \ldots, Z_{1 \mathrm{M}}, Z_{21}, \ldots, Z_{2 \mathrm{M}}, Z_{\mathrm{M} 1}, \ldots\right]$.

$Z_{\mathrm{B} 3}^{\text {пр }}=\left[Z_{12}^{\text {пр }}, \ldots, Z_{1 \mathrm{M}}^{\text {пр }}, Z_{21}^{\text {пр }}, \ldots, Z_{2 \mathrm{M}}^{\text {пр }}, Z_{\mathrm{M} 1}^{\text {пр }}, \ldots\right]$. (30)

Запишем векторы изменения собственных и взаимных комплексных сопротивлений следующим образом:

$$
\begin{aligned}
& \Delta Z_{\text {соб }}=Z_{\text {соб }}-Z_{\text {соб }}^{\text {пр }}, \\
& \Delta Z_{\text {в }}=Z_{\text {в } 3}-Z_{\text {в3 }}^{\text {пр }} .
\end{aligned}
$$


Разделив действительные и мнимые части выражений (31) и (32), для изменения активных и реактивных сопротивлений, получим:

$$
\begin{aligned}
& \Delta R_{\text {соб }}=\operatorname{Re}\left(\Delta Z_{\text {соб }}\right), \\
& \Delta X_{\text {соб }}=\operatorname{Im}\left(\Delta Z_{\text {соб }}\right), \\
& \Delta R_{\text {вз }}=\operatorname{Re}\left(\Delta Z_{\text {вз }}\right), \\
& \Delta X_{\text {в3 }}=\operatorname{Im}\left(\Delta Z_{\text {вз }}\right),
\end{aligned}
$$

Изменение Z-матрицы оценивается евклидовой нормой векторов изменения активного и реактивного сопротивлений [11], т.е.

$$
\begin{aligned}
& \left\|\Delta R_{\text {соб }}\right\|_{2}=\sqrt{\sum_{i, j}^{M}\left|\Delta R_{i i}\right|^{2}}, \\
& \left\|\Delta X_{\text {соб }}\right\|_{2}=\sqrt{\sum_{i, j}^{M}\left|\Delta X_{i i}\right|^{2}}, \\
& \left\|\Delta R_{\mathrm{B} 3}\right\|_{2}=\sqrt{\sum_{i, j}^{M}\left|\Delta R_{i j}\right|^{2}}, \\
& \left\|\Delta X_{\mathrm{B} 3}\right\|_{2}=\sqrt{\sum_{i, j}^{M}\left|\Delta X_{i j}\right|^{2}} .
\end{aligned}
$$

Оценим изменение коэффициентов потерь.Представим матрицу изменения коэффициентов потерь в следующем виде:

$\Delta K=K-K^{\text {пр }}$.

Изменение К-матрицы оценивается евклидовой нормой матрицы потерь [11], т.е.

$$
\|\Delta K\|_{2}=\sqrt{\sum_{i, j}^{M}\left|\Delta K_{i j}\right|^{2}} .
$$

Исследование проводилось для макромодели ЭЭС Армении. Для решении уравнений установившегося режима применяется метод простой итераци. Результаты исследований представлены в таблицах 1-4.

Таблица 1

$Z_{\text {соб- векторы матрицы }}$

\begin{tabular}{|c|c|c|c|c|}
\hline$Z_{\text {соб }}$ & $Z_{11}$ & $Z_{22}$ & $Z_{33}$ & $Z_{44}$ \\
\hline $\begin{array}{c}\text { Значение, } \\
\text { Ом }\end{array}$ & $0.7384+\mathrm{j} 4.1031$ & $.1206+\mathrm{j} 6.1466$ & $0.4986+\mathrm{j} 2.3858$ & $0.6257+\mathrm{j} 3.3042$ \\
\hline$Z_{\text {соб }}^{\text {пр }}$ & $Z_{11}^{\text {пр }}$ & $Z_{22}^{\text {пр }}$ & $Z_{33}^{\text {пр }}$ & $Z_{44}^{\text {пр }}$ \\
\hline $\begin{array}{c}\text { Значение } \\
\text { Ом }\end{array}$ & $0.7334+\mathrm{j} 4.0905$ & $1.1130+\mathrm{j} 6.1270$ & $0.4954+\mathrm{j} 2.3787$ & $0.6210+\mathrm{j} 3.2924$ \\
\hline
\end{tabular}

Таблица 2

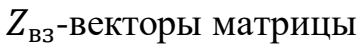

\begin{tabular}{|c|c|c|c|}
\hline$Z_{\text {в3 }}$ & Значение, Ом & $Z_{\text {в3 }}^{\text {пр }}$ & Значение, Ом \\
\hline$Z_{12}$ & $0.6212+\mathrm{j} 3.2827$ & $Z_{12}^{\text {пр }}$ & $0.6153+\mathrm{j} 3.2679$ \\
\hline$Z_{13}$ & $0.5654+\mathrm{j} 2.2003$ & $Z_{13}^{\text {пp }}$ & $0.4615+\mathrm{j} 2.1912$ \\
\hline$Z_{14}$ & $0.6045+\mathrm{j} 3.1721$ & $Z_{14}^{\text {пр }}$ & $0.5997+\mathrm{j} 3.1600$ \\
\hline$Z_{23}$ & $0.4808+\mathrm{j} 2.2833$ & $Z_{23}^{\text {пp }}$ & $0.4762+\mathrm{j} 2.2724$ \\
\hline$Z_{24}$ & $0.6243+\mathrm{j} 3.2916$ & $Z_{24}^{\text {пp }}$ & $0.6185+\mathrm{j} 3.2771$ \\
\hline$Z_{34}$ & $0.4820+\mathrm{j} 2.2920$ & $Z_{34}^{\text {пp }}$ & $0.4785+\mathrm{j} 2.2830$ \\
\hline
\end{tabular}

Таблица 3 


\begin{tabular}{|c|c|c|c|c|}
\hline$K_{i j}$ & 1 & 2 & 3 & 4 \\
\hline 1 & 1.7697 & 2.8402 & -6.8121 & -4.5968 \\
\hline 2 & 2.8402 & 4.5601 & -10.9187 & -7.3682 \\
\hline 3 & -6.8121 & -10.9187 & 26.495 & 17.8758 \\
\hline 4 & -4.5968 & -7.3682 & 17.8758 & 12.0607 \\
\hline
\end{tabular}

Таблица4

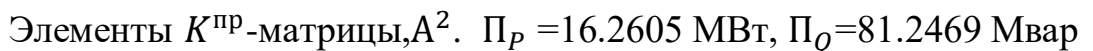

\begin{tabular}{|c|c|c|c|c|}
\hline \multicolumn{1}{|c|}{$K_{i j}^{\text {пр }}$} & 1 & 2 & 3 & 4 \\
\hline 1 & 1.7687 & 2.8391 & -6.8097 & -4.5951 \\
\hline 2 & 2.8391 & 4.5582 & -10.9148 & -7.3654 \\
\hline 3 & -6.8097 & -10.9148 & 26.4869 & 17.8698 \\
\hline 4 & -4.5951 & -7.3654 & 17.8698 & 12.0562 \\
\hline
\end{tabular}

\section{Выводы}

1. Изменение собственных активных и реактивных сопротивлений Z-матрицы, вызванное поперечными пассивными параметрами схемы ЭЭС, составляет: $\left\|\Delta R_{\text {соб }}\right\|_{2}=0.68 \%,\left\|\Delta X_{\text {соб }}\right\|_{2}=0.37 \%$.

2. Изменение взаимных активных и реактивных сопротивлений Z-матрицы вызванное поперечными пассивными параметрами схемы ЭЭС, составляет: $\left\|\Delta R_{\mathrm{B} 3}\right\|_{2}=0.88 \%,\left\|\Delta X_{\mathrm{B} 3}\right\|_{2}=0.42 \%$.

3. Изменение коэффициентов потерь мощности вызванное поперечными пассивными параметрами схемы ЭЭС, составляет: $\|\Delta K\|_{2}=0.03 \%$, изменение потерь активной мощности: $\Delta \Pi_{P}=0.47 \%$, изменение потерь реактивной мощности: $\Delta \Pi_{Q}=0.23 \%$.

4. Полученные новые формулы для потерь мощности позволяют рассчитывать, оценивать иструктурировать потери в питающих сетях по пассивным параметрам схемы системы.

\section{Перспективы дальнейших исследований}

1. Исследование расчета потерь мощности в системообразующих электрических сетях с учетом поперечных пассивных параметров ЛЭП.

2. Разработка новых формул потерь мощности с учетом коэффициентов трансформации

\section{Список бібліографічного опису}

1. Фурсанов М.И. Определение и анализ потерь электроэнергии в электрических сетях энергосистем. Мн.: УВИЦ при УП «Белэнергосбережение», 2005. 208c.

2. Anumaka M.C. Analysis of technical losses in electrical power system (Nigerian 330KV Network as a case study). IJRRAS 2012.12(2). pp.320-327.

3. Zhu Jizhong Optimization of power system operation.New Jersy: John Wiley\&Sons, Inc., Hoboken, 2009.603p.

4. Al-Majeed Ekbal Abd Designing Program for Economic of Operation Power System. Journal of Babylon University. Pure and Applied Sciences. 2011. No (3).Vol. (19). P. 1169-1183.

5. Kothari D.P., Dhillon J.S. Power System optimization.New Delhi: Prentice-Hall of India Private Limited, 2007.572p.

6. Lenin K., Ravindranath Reddy B., and Surya Kalavathi M. Dwindling of real power loss by using Improved Bees Algorithm. International Journal of Recent Research in Electrical and Electronics Engineering (IJRREEE) Vol. 1. Issue 1. P. 34-42. Month: April - June 2014, pp. 34-42.

7. Sarath Babu G., Anupama S., Suresh Babu P. Economic Load Dispatch Solution Including Transmission Losses Using MOPSO. International Journal of Engineering Research and Development. Vol. 9, Iss. 11. P. 15-23.

8. Daniel J.S., Salgado R.S. and Irving M.R. Transmission loss allocation through a modified Ybus. IEE Proc.-Gener. Transm. Distrib. 2005. Vol. 152, No. 2. P.208-214.

9. Аракелян В.П. Оценка падения напряжения в электроэнергетической системе с применением Z- матрицы нового типа. Вчені записки ТНУ імені В.І. Вернадського. Серія: Технічні науки. Київ, 2019. Т.30 (69) Ч. 2 № 4. С.1-5.

10. Аракелян В.П. Новая формула для расчета потерь активной мощности электроэнергетической системы с применением модифицированной Z-матрицы. ВИАА. 2017. Т.14, № 2. С.231-233.

11. Gentle James E. Matrix Algebra. Theory, Computations and Applications in Statistics. Switzerland: Second Edition, Springer Texts in Statistics, 2017. $648 \mathrm{p}$. 


\section{References}

1. Fursanov, M.I. (2005). Determination and analysis of electricity losses in electrical networks of power systems. Minsk: UVITS at UE "Belenergosberezhenie", 208p.

2. Anumaka, M. C. (2012). Analysis of technical losses in electrical power system (Nigerian $330 \mathrm{kV}$ network as a case study). Department Of Electrical Electronic Engineering, Faculty of Engineering, Imo State University, Owerri, Imo State, Nigeria, 320-327.

3. Zhu, J. (2015). Optimization of power system operation. John Wiley \& Sons.

4. Al-Majeed Ekbal Abd (2011). Designing Program for Economic of Operation Power System. Journal of Babylon University/Pure and Applied Sciences, (3)(19), 1169-1183.

5. Kothari D.P., Dhillon J.S. (2007). Power System optimization. New Delhi: Prentice-Hall of India Private Limited.

6. Lenin K., Ravindranath Reddy B., and Surya Kalavathi M. (2014). Dwindling of real power loss by using Improved Bees Algorithm. International Journal of Recent Research in Electrical and Electronics Engineering (IJRREEE) Vol. 1, Issue 1. P. $34-42$.

7. Sarath Babu G., Anupama S., Suresh Babu P. (2014). Economic Load Dispatch Solution Including Transmission Losses Using MOPSO. International Journal of Engineering Research and Development. Vol. 9, Iss. 11. P. 15-23.

8. Daniel J.S., Salgado R.S. Irving M.R. (2005). Transmission loss allocation through a modified Ybus. IEE Proc. Gener. Transm. Distrib.Vol. 152, No. 2. P.208-214.

9. Arakelyan V.P. (2019). Evaluation of the voltage drop in the electric power system using Z-matrices of a new type. Introductory notes from TNU named V.I. Vernadsky. Series: technical sciences, 30 (69), 4, 1-5.

10. Arakelyan V.P. (2017). New formula for calculating active power losses of an electric power system using a modified Z-matrix. VIAA, 14(2), 231-233.

11. Gentle James E. (2017). Matrix Algebra. Theory, Computations and Applications in Statistics. Switzerland: Second Edition, Springer Texts in Statistics. 